Abstract 28 Table 1 The proportion of pupils $(n=106)$ that selected 'Yes' on the questionnaire

\begin{tabular}{llll}
\hline & $\begin{array}{l}\text { Before the Session } \\
\text { (\% of children) }\end{array}$ & $\begin{array}{l}\text { After the Session } \\
\text { (\% of children) }\end{array}$ & $\begin{array}{l}\text { Percentage } \\
\text { Change (\%) }\end{array}$ \\
\hline $\begin{array}{l}\text { Do you know how to } \\
\text { choose healthy foods? }\end{array}$ & 65 & 89 & +24 \\
$\begin{array}{l}\text { Do you know how to read } \\
\text { nutrition labels? }\end{array}$ & 35 & 75 & +40 \\
$\begin{array}{l}\text { Do you know what } \\
\text { diabetes is? }\end{array}$ & 60 & 86 & +26 \\
\hline
\end{tabular}

opportunity to engage with children, practice public speaking skills, and gain confidence.

Conclusions Overall, the feedback received was positive and encouraging. The results show the workshops have successfully engaged with pupils, creating an exciting environment to learn about nutrition. Ultimately, early encouragement of healthy lifestyle choices in childhood may be critical to the development of healthier long-term habits (Llargues et al., 2011). In conclusion, the Food for Thought Project could be an effective school-based intervention to help combat childhood obesity and other lifestyle diseases that may develop later in life.

\section{MULTISYSTEM INFLAMMATORY SYNDROME IN CHILDREN (MIS-C/PIMS): AN OVERVIEW}

Eunice Jia Lin Tang. UK

\subsection{6/bmipo-2021-RCPCH.21}

Background Multisystem inflammatory syndrome in children (MIS-C) or Paediatric inflammatory multisystem syndrome is a newly emerged hyper-inflammatory syndrome that is associated with SARS-CoV-2 infection in the paediatric population.

Objectives We aim to identify commonly presented clinical features, imaging findings, laboratory findings, treatment modalities and clinical outcomes of MIS-C/PIMS. We also aim to compare Kawasaki Disease and MIS-C/PIMS for better identification and management of these patients.

Methods A systematic review was conducted from 1st December 2019 to 30th August 2020. Three medical databases (PubMed, Ovid Resources and the WHO COVID-19 database) were included in this study. Inclusion criteria were all observational studies, case reports or case series that reported data on MIS-C or PIMS.

Results We yielded 48 studies $(\mathrm{N}=1604)$ from 12 countries. Median age ranged from 2 to 19.9 years. $88 \%$ had positive SARS-CoV-2 PCR or serology tests. Reported clinical features include fever (100\%), gastrointestinal symptoms (87\%), rash (56\%), conjunctivitis (48\%) and shock (47\%). ECHO abnormalities $(\mathrm{N}=471)$ were most commonly reported. Laboratory findings include elevated inflammatory markers (66\%-95\%), deranged LFTs (54\%) and cardiac biomarkers (49\%-55\%). Common treatment choices: intravenous immunoglobulin (76\%), steroids (59\%) and aspirin (33\%). ICU admission rate was $72 \%$ and mortality rate was $2 \%$.

Conclusions MIS-C/PIMS is an immune-mediated complication associated with COVID-19. Clinical manifestation vary and majority presents with evidence of multiorgan dysfunction. Close monitoring and high level of care should be given to suspected or confirmed cases. Supportive care remains the mainstay treatment. While manifestation of MIS-C/PIMS overlaps with $\mathrm{KD}$, it is a distinct entity based on their differences in age distribution, geographical and racial variation, laboratory findings, associated features and clinical outcomes.

\section{HIGH FLOW NASAL CANNULA OXYGEN USE IN A DISTRICT GENERAL HOSPITAL IN THE PRE-COVID ERA FROM OCTOBER 2019 TO APRIL 2020}

Laura Johnston, Laura Johnston, Lauren Hetherington, Veena Vasi. UK

\subsection{6/bmjpo-2021-RCPCH.22}

Background High Flow Nasal Cannula (HFNC) oxygen is used to deliver heated and humidified mixture of air and oxygen at a flow higher than the patient's inspiratory flow. It reduces respiratory distress and mouth dryness. The greater oxygen flow 'washes out' the end expiratory oxygen depilated gas, meaning with the next breath, the patient inhales more oxygen. This dead space wash out also reduces $\mathrm{CO} 2$ rebreathing. Reductions in rates of intubation in infants with bronchiolitis have also been reported following introduction of HFNC therapy; however research remains limited within paediatrics and it has been used in patients with conditions, other than bronchiolitis. In our District General Hospital (DGH), we have a guideline for use of HFNC in patients with bronchiolitis but not for use in other respiratory disease. The use of HFNC for the latter group is at individual consultant discretion after discussion with Paediatric Intensive Care Objectives

- What were the incidence, indication, demographics, duration and outcome of patients who received HFNC therapy on the Children's ward in Craigavon Hospital over a 6 month period from October 2019 to March 2020?

- When patients received HFNC therapy outside our current indication, were they discussed with PICU in terms of suitability to initiate or continue therapy in the children's ward?

- Was there a delay or perceived delay in escalation of treatment to tracheal intubation in those who had treatment failure on HFNC?

Methods This was a retrospective chart review. Patient demographics, indication, duration of treatment and outcome are recorded in a pre-designed proforma

SHSCT Research Governance team advised this is a service evaluation quality improvement which did not require ethics approval (Appendix 2).

Treatment success was defined as 'patient improving on HFNC with successful weaning of respiratory support.' Treatment failure was described as the 'patient deteriorating despite treatment and requiring admission to PICU'

Results are tabulated in XCEL format, analysed and conclusions drawn with recommendations

Results

- 28 children required HFNC therapy over a 6 month period with peak activity in November 2019

- Male to female ratio was 3.5:1

- Commonest indication for use (85\% cases) was bronchiolitis

- Comorbidities were seen in 50\% of the cases with commonest being prematurity

- All patients receiving HFNC outside agreed indications were appropriately discussed with PICU 